\title{
Robust Video Data Hiding using Forbidden Zone Data Hiding and Selective Embedding
}

\author{
Kundalakesi $^{1}$, Abarna $^{2}$, Kalaiselvi ${ }^{3}$, Sushmitha ${ }^{4}$ \\ Assistant Professor, Computer Application and Software Systems, Sri Krishna Arts and Science College,
} Coimbatore, India ${ }^{1}$

Student, Computer Application and Software Systems, Sri Krishna Arts and Science College, Coimbatore, India ${ }^{2,3,4}$

\begin{abstract}
Now a day, it is very risky to handle the data in Internet against intruders the peoples invented a large thing to protect the data and there are lots of hidings techniques are to be invented for security purpose. But those techniques can be hack by unauthorized users is drawback in existing systems so that propose the new system i.e. Data hiding behind the video using forbidden zone and selective embedding. This system makes use of correction ability of duplication store codes and advantage of forbidden zone data hiding is used. This system is tested by all types of videos that type of video which help to data hiding likewise avi, 3gp, mp4 etc. In this research the encryption and decryption technique is used to provide the security key. Without that key no one can see the original data. This technique is used to protect the database from unauthorized and the destructive forces .It has large erasure capability of data hiding.
\end{abstract}

Keywords: Steganography, Data Hiding, Forbidden Zone, Selective embedding.

\section{INTRODUCTION}

Steganography is a Greek word which means "covered or hidden writing". The idea of steganography is thousands of years old. Data hiding can be used for secret transmission. Steganography is a technique for hiding secret information in digital image, audio and video to secure information from third party .The capacity of steganography is in hiding the private data by indistinct, hiding its existence in a non-secret carrier file. In this sense, steganography is differing from cryptography, which involves creating the content of the secret information unreadable while not stopping non-intended viewers from learning about its existence.

Steganography apply in various fields such as military and industrial applications. Lossless steganography techniques are use for secure and successful transmission of information from sender to receiver. Usually, steganography was based on hiding secret message in digital image files. Recently, the computer programmers start interest applying steganographic techniques to video files as well as audio files

This time multimedia objects like image, audio, video are used as a cover media by steganographic systems because public often send digital pictures in email and other Internet communication. The image file formats are JPEG, GIF, BMP, audio file formats are WAV, MP3, and video file formats are MPEG, MP4, and AVI.

\section{EXISTING SYSTEM}

In special domain, the hiding process such as least significant bit (LSB) replacement is done in special domain, while transform domain methods; hide data in another domain such as wavelet domain. Least significant bit (LSB) is the simplest form of Steganography. LSB is based on inserting data in the least significant bit of pixels, which lead to a slight change

On the cover image that is not noticeable to human eye. Since this method can be easily cracked, it is more vulnerable to attacks.

LSB method has intense affects on the statistical information of image like histogram. Attackers could be aware of a hidden communication by just checking the Histogram of an image. A good solution to eliminate this defect was LSB matching. LSBMatching was a great step forward in Steganography methods and many others get ideas from it

\section{PROPOSED SYSTEM}

Data hiding in video sequences is performed in two major ways: bit stream-level and data-level.

In this paper, we propose a new block-based selective embedding type data hiding framework that encapsulates Forbidden Zone Data Hiding (FZDH)

By means of simple rules applied to the frame markers, we introduce certain level of robustness against frame drop, repeat and insert attacks.

\section{ADVANTAGES}

User cannot find the original data.

It is not easily cracked.

To increase the Security.

To increase the size of stored data.

We can hide more than one bit. 
Vol. 4, Issue 1, January 2017

\section{SYSTEM ARCHITECTURE}

A system design or systems planning is the abstract model that defines the structure, activities, and more visions of a system. An architecture explanation is a formal explanation and representation of a system, organized in a way that supports reasoning about the structures of the system. A system architecture of "Hiding Text in video using FZDH and selective Embedding Process i.e. encode and Extracting Process i.e. Decode can comprise system components, the externally observable properties of those modules, the relationships between them. The main focus of the proposed system architecture is to achieve provide better security for the sharing of data. The proposed system architecture is shown in below figure

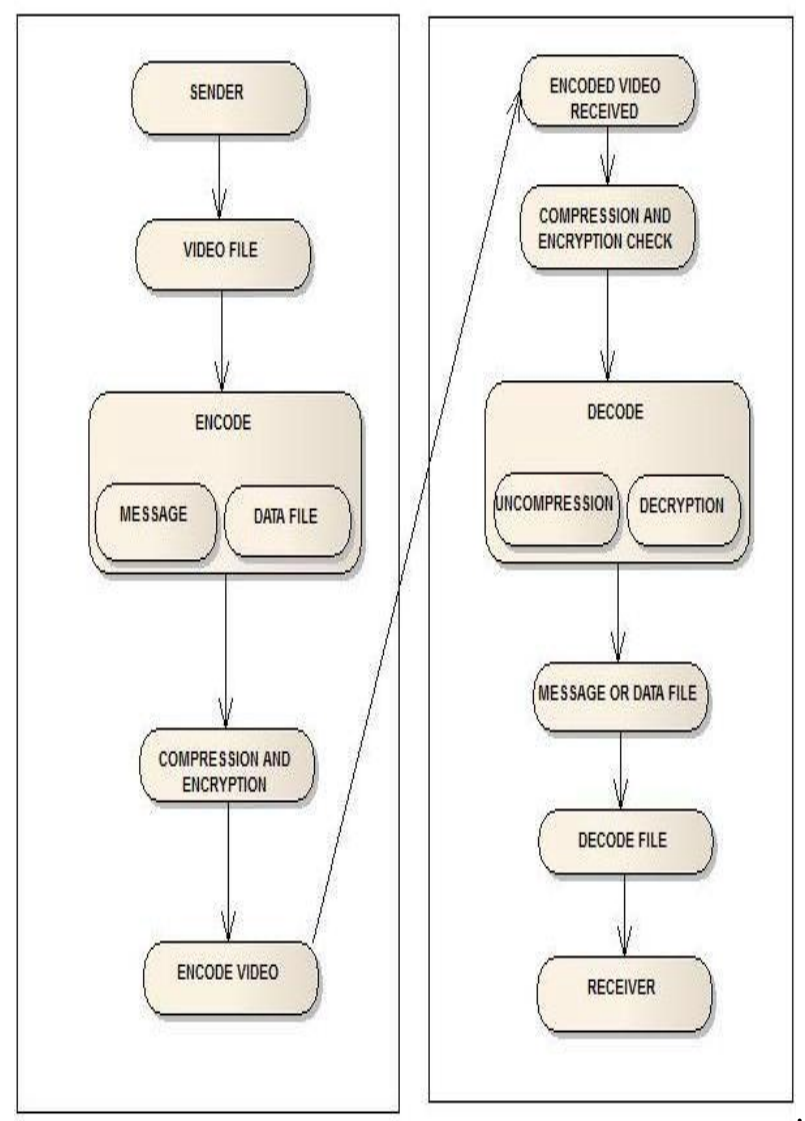

Fig 1. System Architecture Block Diagram

\section{FRAMEWORK}

\section{A. SELECTIVE EMBEDDING:}

Host signal models, which will be used in data hiding, are single-minded adaptively. The selection is performed at four stages: border variation, frequency band willpower, block selection, and coefficient selection

\section{B. FRAME SELECTION:}

A number of numbers of blocks in the whole frame is calculated. If the percentage of selected blocks to all blocks is above a certain value (T0) the frame is handled. Otherwise, this frame is avoided.

\section{FREQUENCY BAND:}

Only certain DCT constants are operated. Middle frequency band of DCT constants.

\section{BLOCK SELECTION:}

Energy of the constants in the mask is added. If the vitality of the block is overhead an assured value (T1) then the block is managed. Otherwise, it is avoided.

\section{E. COEFFICIENTS SELECTION:}

Energy of each constant is compared to another beginning T2. If the energy is above T2, then it is used during data inserting together with other selected constants the same block.

\section{F. BLOCK PARTITIONING:}

Two split data sets are inserted; message bits (m1) and frame synchronization markers $(\mathrm{m} 2)$. The block locations of $\mathrm{m} 2$ are resolute randomly depending on a random key. The rest of the blocks are kept for $\mathrm{m} 1$. The same splitting is used for all frames. $\mathrm{m} 2$ is inserted frame by frame. Continuously the other hand, $\mathrm{m} 1$ is single to $\mathrm{T}$ sequential frames. Both of them are found as the outcomes of the RA encoder.

\section{G. ERASURE HANDLING:}

Due to adaptive block collection, de-synchronization occurs between embedded and translator. As a result of attacks or even embedding operation decoder may not perfectly determine the selected blocks at the embedded. In order to overcome this problem, mistake modification codes strong to erasures, such as RA codes are used in video data hiding in previous hard work. RA code is a low complication turbo-like code. It is collected of repetition code, interleave, and a convolution encoder. The source bits $(\mathrm{u})$ are repetitive $\mathrm{R}$ times and accidentally permuted dependent on a key. The interleaved sequence is passed through a convolution encoder with a transfer function 1/ $(1+D)$, where D represents a _rst-order stay. In efficient RA code.

\section{SYSTEM WORKING}

There are two Modules are used in this project:

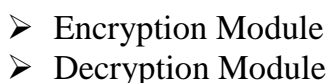

\section{ENCRYPTION MODULE}

In Encryption segment, it contains of Key file part and video and data or data file, the video can be browse using browse control, before the user can style the data or else can upload the data also though the look control, when it is clicked the open file dialog box is opened and where the user can select the secret note. The frames are selected for given message or file, where key file can be specified with the password as a different security in it. Then the user can clicked on Encrypt video button. Then the data file or message is hidden in video using Forbidden Zone Data Hiding Technique. 


\section{DECRYPTION MODULE}

This module is the reverse as such as Encryption segment. The video used in Encryption can be browse using browse control, where the Key file should be also definite similar as that of encryption portion. Then the user can clicked on decryption control, and then the secreted message is presented in the text zone definite in the application or else it is take out to the place where the user specified it.

\section{CONCLUSION}

A video data hiding approach in which the host signal which can be used for data hiding is selected by selective embedding and forbidden zone concept. In addition to this we use the properties of human visual system. A piecewise mapping function according to human visual sensitivity of contrast is used so that adaptively can be achieved without extra bits for overhead. Video data hiding in human visual system is an approach to hide the data in a video in a secure way by using the concepts in human visual system.

\section{REFERENCES}

[1] Channalli S, Jadhav A (2009) Steganography an art of hiding data. Int J Computer Science Eng 1(3):137-141

[2] http://www.slideshare.net/pnlakha/robust-video-data-hiding-usingforbidden-zone-data-hiding-and-selective-embedding-22898600

[3] http://ieeexplore.ieee.org/document/7494111/?reload=true https://www.researchgate.net/publication/292206033_Robust_video _steganography_algorithm_using_adaptive_skin-tone_detection

[4] http://www.slideshare.net/madonnaranjini/robust-video-datahiding-using-forbidden-zone

[5] http://www.engpaper.net/video-steganography-engineeringresearch-papers.html 